\title{
An Algebraic Method for Harmonic Responses based on Extended Hybrid Expansion Method
}

\author{
Jingfang Shen ${ }^{1, a}$ and Wenlin Huang ${ }^{1, b^{*}}$ \\ ${ }^{1}$ School of Science, Huazhong Agricultural University, Wuhan 430070, China \\ ashenjingfang16@126.com, bhwl_19961029@126.com
}

\begin{abstract}
Keywords: Harmonic responses analysis; Modal superposition; Modal truncation error; Extended hybrid expansion method
\end{abstract}

\begin{abstract}
An algebraic method is presented for harmonic responses analysis. This paper is aiming at improving the accuracy of results obtained by the extended hybrid expansion method. Based on modal superposition and Neumann expansion theorem, the high and low modal can be truncated by the proposed method. Meanwhile, how to reduce the truncation error is also our pivotal topic. According to the accuracy of the results, two methods have been compared in several different situations. In the end, the results of simulation experiments show clearly the correctness and effectiveness of the improved method.
\end{abstract}

\section{Introduction}

Harmonic response analysis is widely used in mechanical systems. Often, a lot of rolling machine parts such as blowers, engines and boosters may cause oscillatory excitation [1]. Therefore, harmonic response analysis is playing an increasingly important role in many areas. For example, it can be used to identify the system, control the noise, update the finite element model, detect the structural damage and so on.

In the past twenty years, a lot of methods have been used in harmonic response analysis and the sensitivity of non-viscously damped systems. First of all, harmonic responses are usually calculated by direct frequency response method (DFRM) and modal superposition method (MDM). From the direct frequency results, the DFRM uses the complicated arithmetic to solve the equation of motion. Hence, the results may be calculated accurately. The MDM expresses harmonic responses as a combination of low, high and middle modes. These methods are widely applied in engineering field and have been implemented in utility software. As can be seen, the MDM needs all the modes to calculate the responses, nevertheless, it is usually hard and even dispensable to obtain all the modes in engineering. Therefore, it often calculates the responses by using the interest modes and the modal truncation error is introduced. In order to eliminate the modal truncation error, many researchers have investigated the corrections to the modal truncation scheme. Then, modal acceleration methods (MAM) [2] is introduced by Maddox, Hansteen and Bell, Leger and Wilson, Kulkarni and Ng. Dickens. The characteristic method uses the particular solution of the dynamic equation that the excitation frequency is set to zero, meanwhile, it combines the particular solution with the mode superposition of available modes. Due to its neglect for the acceleration terms of the responses, the approximate results are usually obtained by using the method. Through further study, hybrid methods[3] eliminate the error by expressing the responses as a power-series expansion of dynamic response in terms of system matrices. Dynamic correction methods [4] reduce the caculated error by including the contribution of motion and the high-order modes by a summation of the particular solutions of both the equation of motion and the reduced differential equation of motion. 
It is worth mentioning that force derivative methods[5] which consider the higher-order derivatives of the forcing function reduce the error.

In this article, we aim at accurately calculating the harmonic response of non-viscously damped systems in terms of the modes of interest by generalizing the extended hybrid expansion method (EHEM). The extended hybrid expansion method(EHEM) is presented to reduce the error of the results obtained by the MDM. We generalize the extended hybrid expansion method (EHEM) by multiplying two coefficients in the two terms respectively. So that, the EHEM is a particular situation. By adjusting the coefficients, it may obtain various methods to calculate harmonic responses. In this way, the error can be reduced efficiently.

\section{Theoretical Background}

In engineering, the equations of motion of a viscously damped system can be expressed as

$$
\left(-\omega^{2} M+i \omega C+K\right) X(i \omega)=F_{h} .
$$

Where $\mathrm{M}$ denotes the mass, $\mathrm{C}$ denotes viscous damping and $\mathrm{K}$ denotes stiffness matrices. Here, we only consider system matrices are symmetric.

Assuming the complex eigenvalues are different, the complex frequency response function (FRF) matrix $A(i \omega)$ can be expressed by mode superposition

$$
A(i \omega)=\sum_{j=1}^{2 N} \frac{\varphi_{j} \varphi_{j}^{T}}{\theta_{j}\left(i \omega-\lambda_{j}\right)} \quad \text { with } \quad \theta_{j}=\varphi_{j}^{T}\left(2 \lambda_{j} M+C\right) \varphi_{j}
$$

Where $\varphi_{j}$ is the eigenvector corresponding to the jth eigenvalue $\lambda_{j}$. Here, we assume the number of eigenvalues is $2 \mathrm{~N}$.

By considering $A(i \omega)=\left(-\omega^{2} M+i \omega C+K\right)^{-1}$, the responses from Eq.1 can be expressed as

$$
X(i \omega)=\sum_{j=1}^{2 N} \frac{\varphi_{j}^{T} F_{h} \varphi_{j}}{\theta_{j}\left(i \omega-\lambda_{j}\right)}
$$

In theory, the MDM needs all the frequencies and mode shapes. However, often the $L_{1}$ th pair through $L_{2} t h$ pair of complex modes lie in the frequency range of interest and only these modes of interest are calculated. So, the harmonic responses obtained using the MDM can be expressed by

$$
X_{M D M}^{M}(i \omega)=\sum_{j=2 L_{1}}^{2 L_{2}} \frac{\varphi_{j}^{T} F_{h} \varphi_{j}}{\theta_{j}\left(i \omega-\lambda_{j}\right)} .
$$

The corresponding modal truncation error can be given by

$$
E_{M D M}^{M}(i \omega)=\sum_{j=1}^{2 L_{1}-1} \frac{\varphi_{j}^{T} F_{h} \varphi_{j}}{\theta_{j}\left(i \omega-\lambda_{j}\right)}+\sum_{j=2 L_{2}+1}^{2 N} \frac{\varphi_{j}^{T} F_{h} \varphi_{j}}{\theta_{j}\left(i \omega-\lambda_{j}\right)} .
$$


Accurate modal correction method

If the excitation frequencies $\omega_{\text {satisfy the inequation }}$

$\left|\frac{i(\omega-\sigma)}{\lambda_{j}-i \sigma}\right|<1, \forall j=1,2, \cdots, 2 N$

where $\sigma$ is a frequency shift value, then the FRF matrix can be expressed as

$$
A(i \omega)=-\sum_{k=0}^{\infty} \sum_{j=1}^{2 N}[i(\omega-\sigma)]^{k} \frac{\varphi_{j} \varphi_{j}^{T}}{\theta_{j}\left(\lambda_{j}-i \sigma\right)^{k+1}} .
$$

By casting Eq.6 into the matrix form, we obtain

$$
A(i \omega)=-\sum_{r=1}^{\infty}[i(\omega-\sigma)]^{r-1} U \Theta^{-1} \Lambda^{-r} U^{T}
$$

where $\Lambda=\operatorname{diag}\left[\lambda_{1}-i \sigma, \lambda_{2}-i \sigma, \cdots, \lambda_{2 N}-i \sigma\right], U=\left[\varphi_{1}, \varphi_{2}, \cdots, \varphi_{2 n}\right]$ and $\Theta=\operatorname{diag}\left[\theta_{1}, \theta_{2}, \cdots, \theta_{2 N}\right]$.

By using the relationship expressed in Eq.1, the responses can be obtained as the following form

$$
X(i \omega)=-\sum_{k=0}^{\infty} \sum_{j=1}^{2 N}[i(\omega-\sigma)]^{k} \frac{\varphi_{j} F_{h} \varphi_{j}^{T}}{\theta_{j}\left(\lambda_{j}-i \sigma\right)^{k+1}} .
$$

The harmonic responses obtained by MDM can be given by

$$
X_{M D M}^{M}(i \omega)=-\sum_{k=0}^{\infty} \sum_{j=2 L_{1}}^{2 L_{2}}[i(\omega-\sigma)]^{k} \frac{\varphi_{j} F_{h} \varphi_{j}^{T}}{\theta_{j}\left(\lambda_{j}-i \sigma\right)^{k+1}} .
$$

the mode truncation error expressed as

$$
E_{M D M}^{M}(i \omega)=-\sum_{k=0}^{\infty}[i(\omega-\sigma)]^{k}\left[\sum_{j=1}^{2 L_{1}-1} \frac{\varphi_{j}^{T} F_{h} \varphi_{j}}{\theta_{j}\left(i \omega-\lambda_{j}\right)}+\sum_{j=2 L_{2}+1}^{2 N} \frac{\varphi_{j}^{T} F_{h} \varphi_{j}}{\theta_{j}\left(i \omega-\lambda_{j}\right)}\right] .
$$

For a viscously damped systems, the eigensolutions and system matrices satisfy

$$
-\sum_{j=1}^{2 N} \frac{\varphi_{j} \varphi_{j}^{T}}{\theta_{j}\left(\lambda_{j}-i \sigma\right)^{k+1}}=\overline{\Gamma_{k}}, \forall k=0,1,2, \cdots \infty
$$

Where

$$
\left\{\begin{array}{l}
\overline{\Gamma_{0}}=\left(K+i \sigma C-\sigma^{2} M\right)^{-1} \\
\overline{\Gamma_{1}}=-\left(K+i \sigma C-\sigma^{2} M\right)^{-1}(C+i 2 \sigma M) \times\left(K+i \sigma C-\sigma^{2} M\right)^{-1} \\
\overline{\Gamma_{k}}=-\left(K+i \sigma C-\sigma^{2} M\right)^{-1}(C+i 2 \sigma M) \overline{\Gamma_{K-1}}-\left(K+i \sigma C-\sigma^{2} M\right)^{-1} M \overline{\Gamma_{k-2}} .
\end{array}\right.
$$

By casting Eq.11 into the matrix form, one obtains

$$
-U \Theta^{-1} \Lambda^{-k-1} U^{T}=\overline{\Gamma_{k}}, \forall k=0,1,2, \cdots \infty .
$$


where $\Lambda=\operatorname{diag}\left[\lambda_{1}-i \sigma, \lambda_{2}-i \sigma, \cdots, \lambda_{2 N}-i \sigma\right], U=\left[\varphi_{1}, \varphi_{2}, \cdots, \varphi_{2 n}\right]$ and

$\Theta=\operatorname{diag}\left[\theta_{1}, \theta_{2}, \cdots, \theta_{2 N}\right]$

By using the results expressed in Eq.11, the mode truncation error expressed in Eq.10 can be expressed as

$$
E_{M D M}^{M}(i \omega)=\sum_{k=0}^{\infty}[i(\omega-\sigma)]^{k}\left[\overline{\Gamma_{k}} F_{h}+\sum_{j=2 L_{1}}^{2 L_{2}} \frac{\varphi_{j}^{T} F_{h} \varphi_{j}}{\theta_{j}\left(\lambda_{j}-i \sigma\right)^{k+1}}\right] .
$$

Adding the error term to the MDM in Eq.4, the EHEM takes the form

$$
X_{E H E M}(i \omega)=\sum_{j=2 L_{1}}^{2 L_{2}} \frac{\varphi_{j}^{T} F_{h} \varphi_{j}}{\theta_{j}\left(i \omega-\lambda_{j}\right)}+\sum_{k=0}^{\infty}[i(\omega-\sigma)]^{k}\left[\overline{\Gamma_{k}} F_{h}+\sum_{j=2 L_{1}}^{2 L_{2}} \frac{\varphi_{j}^{T} F_{h} \varphi_{j}}{\theta_{j}\left(\lambda_{j}-i \sigma\right)^{k+1}}\right] .
$$

In practice, it is hard to obtain all terms on the right-hand of Eq.15. Therefore, we consider the first $\mathrm{h}$ terms are retained, the approximate responses by the EHEM can easily be obtained as

$$
X_{E H E M}(i \omega)=\sum_{j=2 L_{1}}^{2 L_{2}} \frac{\varphi_{j}^{T} F_{h} \varphi_{j}}{\theta_{j}\left(i \omega-\lambda_{j}\right)}+\sum_{k=0}^{h-1}[i(\omega-\sigma)]^{k}\left[\overline{\Gamma_{k}} F_{h}+\sum_{j=2 L_{1}}^{2 L_{2}} \frac{\varphi_{j}^{T} F_{h} \varphi_{j}}{\theta_{j}\left(\lambda_{j}-i \sigma\right)^{k+1}}\right] .
$$

Because only the first $\mathrm{h}$ term are retained, there also exists truncation error in Eq.16. It can be expressed as

$$
E_{E H E M}=\sum_{k=h}^{\infty}[i(\omega-\sigma)]^{k}\left[\overline{\Gamma_{k}} F_{h}+\sum_{j=2 L_{1}}^{2 L_{2}} \frac{\varphi_{j}^{T} F_{h} \varphi_{j}}{\theta_{j}\left(\lambda_{j}-i \sigma\right)^{k+1}}\right]
$$

\section{Modal Truncation Augmentation Method}

Owing to the fact that EHEM only uses the first $\mathrm{h}$ terms of the power-series expansion expressed in Eq.15, the actual remaining error of the EHEM may be large.

The modal truncation augmentation method MTAM is therefore presented to improve the results obtained using EHEM by multiplying two coefficients in the two terms respectively in the EHEM. The responses calculated using the MTAM can be expressed as

$$
X_{E H E M}^{M T A M}(i \omega)=\alpha(i \omega) \sum_{j=2 L_{1}}^{2 L_{2}} \frac{\varphi_{j}^{T} F_{h} \varphi_{j}}{\theta_{j}\left(i \omega-\lambda_{j}\right)}+\beta(i \omega) \sum_{k=0}^{\infty}[i(\omega-\sigma)]^{k}\left[\overline{\Gamma_{k}} F_{h}+\sum_{j=2 L_{1}}^{2 L_{2}} \frac{\varphi_{j}^{T} F_{h} \varphi_{j}}{\theta_{j}\left(\lambda_{j}-i \sigma\right)^{k+1}}\right]
$$

Where $\alpha(i \omega)$ and $\beta(i \omega)$ are undetermined functions of Laplace varibles.Consider the first $\mathrm{h}$ terms are retained, the results calculated can be expressed as

$$
X_{E H E M}^{M T A M}(i \omega)=\alpha(i \omega) \sum_{j=2 L_{1}}^{2 L_{2}} \frac{\varphi_{j}^{T} F_{h} \varphi_{j}}{\theta_{j}\left(i \omega-\lambda_{j}\right)}+\beta(i \omega) \sum_{k=0}^{h-1}[i(\omega-\sigma)]^{k}\left[\overline{\Gamma_{k}} F_{h}+\sum_{j=2 L_{1}}^{2 L_{2}} \frac{\varphi_{j}^{T} F_{h} \varphi_{j}}{\theta_{j}\left(\lambda_{j}-i \sigma\right)^{k+1}}\right]
$$

The corresponding error can be expressed as 


$$
\begin{aligned}
E_{\text {EHEM }}^{\text {MTAM }}(i \omega)= & {[1-\alpha(i \omega)] \sum_{j=2 L_{1}}^{2 L_{2}} \frac{\boldsymbol{\varphi}_{j}^{T} F_{h} \boldsymbol{\varphi}_{j}}{\boldsymbol{\theta}_{j}\left(i \omega-\lambda_{j}\right)}+[1-\beta(i \omega)] \sum_{k=0}^{h-1}[i(\omega-\sigma)]^{k}\left[\overline{\Gamma_{k}} F_{h}+\sum_{j=2 L_{1}}^{2 L_{2}} \frac{\boldsymbol{\varphi}_{j}^{T} F_{h} \boldsymbol{\varphi}_{j}}{\left.\lambda_{j}-i \boldsymbol{\sigma}\right)^{k+1}}\right] } \\
& +\sum_{k=h}^{\infty}[i(\omega-\sigma)]^{k}\left[\overline{\Gamma_{k}} F_{h}+\sum_{j=2 L_{1}}^{2 L_{2}} \frac{\boldsymbol{\varphi}_{j}^{T} F_{h} \boldsymbol{\varphi}_{j}}{\left.\boldsymbol{\theta}_{j}-i \boldsymbol{\sigma}\right)^{k+1}}\right]
\end{aligned}
$$

For convenience, we define the difference of the two method as

$$
S(i \omega)=E_{E H E M}^{M T A M}(i \omega)-E_{E H E M}(i \omega)
$$

$S(i \omega)$ can be expressed as

$$
S(i \omega)=[1-\alpha(i \omega)] \sum_{j=2 L_{1}}^{2 L_{2}} \frac{\varphi_{j}^{T} F_{h} \varphi_{j}}{\theta_{j}\left(i \omega-\lambda_{j}\right)}+[1-\beta(i \omega)] \sum_{k=0}^{h-1}[i(\omega-\sigma)]^{k}\left[\overline{\Gamma_{k}} F_{h}+\sum_{j=2 L_{1}}^{2 L_{2}} \frac{\varphi_{j}^{T} F_{h} \varphi_{j}}{\theta_{j}\left(\lambda_{j}-i \sigma\right)^{k+1}}\right]
$$

By casting Eq.4 and Eq.16 into the Eq.21, one obtains

$$
S(i \omega)=[1-\alpha(i \omega)] X_{M D M}^{M}(i \omega)+[1-\beta(i \omega)]\left[X_{E H E M}(i \omega)-X_{M D M}^{M}(i \omega)\right]
$$

If $\alpha(i \omega)$ and $\beta(i \omega)$ satisfy the following condition:

$$
\alpha(i \omega)>\left[X_{E H E M}(i \omega)-\beta(i \omega) X_{E H E M}(i \omega)+\beta(i \omega) X_{M D M}^{M}(i \omega)\right] \cdot\left[X_{M D M}^{M}(i \omega)\right]^{-1}
$$

The difference of the two method $S(i \omega)_{<0}$, in that case Modal truncation augmentation method is efficient in decreasing the calculated error. Especially, $S(i \omega)$ can be expressed as

$$
S(i \omega)=\left\{\begin{array}{l}
0, \alpha(i \omega)=\beta(i \omega)=1 \\
X_{M D M}^{M}(i \omega), \alpha(i \omega)=0, \beta(i \omega)=1 \\
X_{E H E M}(i \omega)-X_{M D M}^{M}(i \omega), \alpha(i \omega)=1, \beta(i \omega)=0 \\
\frac{1}{2} X_{E H E M}(i \omega), \alpha(i \omega)=\beta(i \omega)=\frac{1}{2}
\end{array} .\right.
$$

\section{Example Study}

To compare the accuracy of the two method, we respectively computed the amplitude of one frequency response function of the different methods when $\mathrm{h}=1$. At the same time, three different situations are also obtained. The results obtained are shown below. 


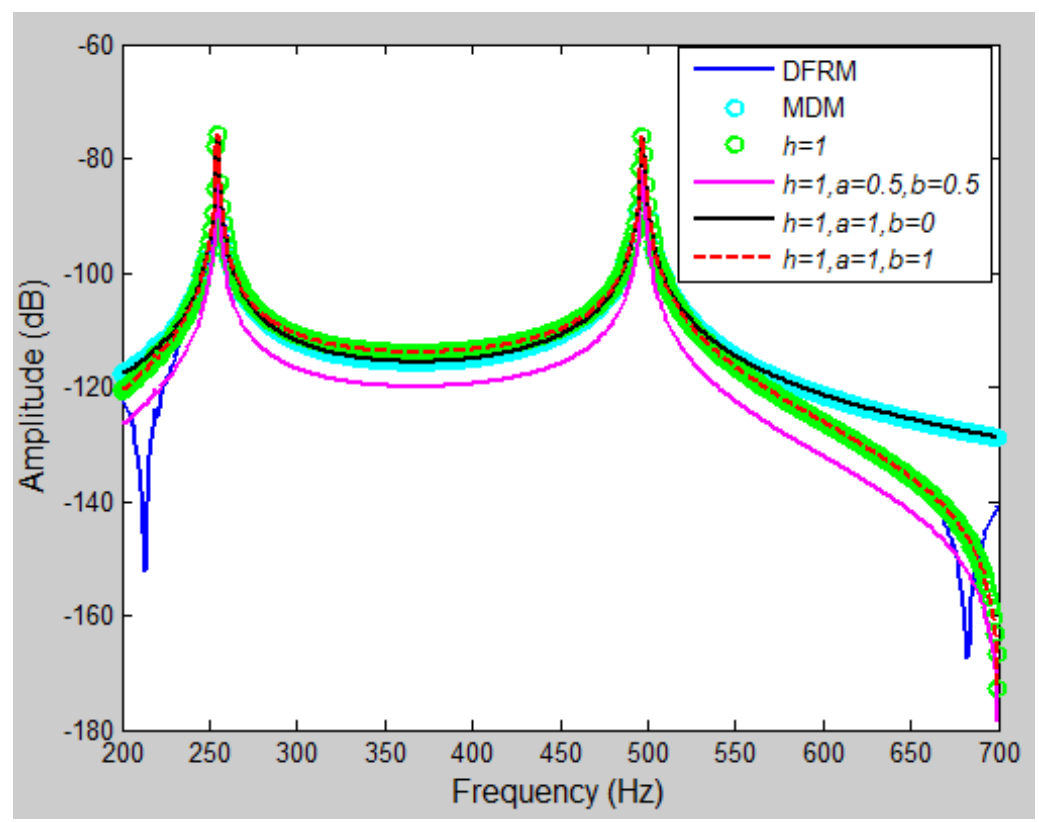

Figure 1. the amplitude of one frequency response function of the different methods

From the result in Fig. 1 the errors of the MDM may be obvious when the frequency locates in $200-250 \mathrm{~Hz}$ and $650-700 \mathrm{~Hz}$. When $\alpha(i \omega)=1, \beta(i \omega)=0$, in that case, the improved method MTAM is equivalent to the MDM. When $\alpha(i \omega)=1, \beta(i \omega)=1$, the improved method MTAM is equivalent to the EHEM. Apparently, when $\alpha(i \omega)=0.5, \beta(i \omega)=0.5$, the improved method MTAM can reduce the error of MDM and EHEM when the frequency locates in $200-250 \mathrm{~Hz}$ and $650-700 \mathrm{~Hz}$.

\section{Concluding Remarks}

The harmonic response analysis is widely applied in many areas. Owing to its difficulty to obtain all the modes of a model, there exists the modal truncation error when computing the frequency response function. The paper is aimed at improving the accuracy of results obtained by the extended hybrid expansion method. The modal truncation augmentation method MTAM is therefore presented to improve the result obtained by EHEM. The MTAM generalizes the extended hybrid expansion method (EHEM) by multiplying two coefficients in the two terms respectively in the EHEM. According to the experiments, the errors of the MDM may be obvious when the frequency locates in $200-250 \mathrm{~Hz}$ and $650-700 \mathrm{~Hz}$. When $\alpha(i \omega)=1, \beta(i \omega)=0$, in that case, the improved method MTAM is equivalent to the MDM. When $\alpha(i \omega)=1, \beta(i \omega)=1$, the improved method MTAM is equivalent to the EHEM. Apparently, when $\alpha(i \omega)=0.5, \beta(i \omega)=0.5$, the improved method MTAM can reduce the error of MDM and EHEM when the frequency locates in $200-250 \mathrm{~Hz}$ and $650-700 \mathrm{~Hz}$. 


\section{Acknowledgement}

This work was supported by National university students innovation project(201610504076) and Higher school university mathematics teaching research and development center project(CMC20160408). Thanks to Wenlin Huang who is the corresponding author.

\section{References}

[1] L. Li, Y.J. Hu, X.L. Wang, Accurate method for harmonic responses of non-classically damped systems in the middle frequency range, J. Sound Vibr.(2014)1-16.

[2] J.M. Dickens, J.M. Nakagawa, M.J. Wittbrodt, A critique of mode acceleration and modal augmentation methods for modal response analysis, Computers and Structures 62(1997)985-998.

[3] Qu Z-Q, Selvam RP, Hybrid expansion method for frequency responses and their sensitivities, Journal of Sound and Vibration 238(2000)369-388.

[4] G. Borino, G. Muscolino, Mode-superposition methods in dynamic analysis of classically and non-classically damped linear systems. Earthquake Engineering and Structural Dynamic 14(1986)705-717.

[5] M.A. Akgun, A new family of mode-superposition methods for response calculations, Journal of Sound and Vibration 167(1993) 289-302.

[6] L. Li, Y.J. Hu, X.L. Wang, L Lu, A hybrid expansion method for frequency response functions of non-proportionally damped systems, Mech. Syst. Signal Process.42(2014)31-41.

[7] L Li, Y.J. Hu, X.L.Wang, Improved approximate methods for calculating frequency response function matrix and response of MDOF system with viscoelastic hereditary terms, J. Sound Vibr. 332(2013)3945-3956.

[8] L. Li, Y.J. Hu, X.L. Wang, Eliminating the modal truncation problem encountered in frequency responses of viscoelastic systems, J. Sound Vibr. 333(2014)1182-1192.

[9] L. Li, Y.J. Hu, X.L. Wang, Design sensitivity analysis of dynamic response of nonviscously damped systems, Mech. Syst. Signal Process.41(2013)613-638.

[10]A Palmeri, G Muscolino, A numerical method for the time-domain dynamic analysis of buildings equipped with viscoelastic dampers, Struct. Control Health Monit. 18(2011)519-539. 